\title{
Magnesium Acceptor Energy Levels in Cubic GaN
}

\author{
H. Przybylińska, R. Buczko \\ Institute of Physics, Polish Academy of Sciences \\ al. Lotników 32/46, 02-668 Warsaw, Poland \\ G. Kocher and W. Jantsch \\ Institute of Semiconductor and Solid State Physics, Johannes Kepler University \\ Linz, Austria \begin{abstract}
measured with the use of photothermal ionization spectroscopy. Apart from the photoionization band several sharp features were detected, related to internal $\mathrm{Mg}$-acceptor transitions. The transitions were identified with the help of effective-mass model calculations involving light- and heavy-hole as well as spin-orbit split-off bands. Transitions to resonant states, associated with the spin-orbit split-off valence band, were also identified. The determined hole binding energy of the $\mathrm{Mg}$ acceptor in zinc-blende GaN is $236 \pm 1 \mathrm{meV}$.
\end{abstract} \\ Intra-impurity transitions of the $\mathrm{Mg}$ acceptor in cubic phase GaN were
}

PACS numbers: 78.30.-j, 71.55.-i, 71.55.Eq

\section{Introduction}

Despite the rapid technological progress achieved in the development of GaN based devices p-type doping of GaN still remains an important issue. Magnesium is the most commonly used impurity for $p$-type doping of GaN, however, the hole binding energy of this acceptor was never directly measured and only estimated from photoluminescence or electrical experiments. The experimental data obtained from the Hall effect measurements yield a wide range of thermal activation energies depending on the concentration and degree of compensation, while the interpretation of the results of optical studies, such as donor-to-acceptor transitions, require assumptions regarding the donor involved (for a review see Ref. [1]). In this paper we report direct measurements of internal Mg-related transitions with the use of photothermal ionization spectroscopy. The transitions were identified with the help of effective-mass model calculations. 


\section{Experimental results}

The samples investigated were $490 \mathrm{~nm}$ thick, cubic $\mathrm{GaN}: \mathrm{Mg}$ films grown on semi-insulating GaAs(001) substrates by rf plasma-assisted molecular beam epitaxy. The room temperature hole concentration was $1.4 \times 10^{16} \mathrm{~cm}^{-3}$. Photoconductivity was measured with the use of a BOMEM DA8.23 Fourier transform spectrometer in the spectral range of $50-500 \mathrm{meV}$. The sample was placed on a cold finger of a Cryoflow cryostat in an external sample chamber. The sample was illuminated with the $\mathrm{KBr}$ split beam of the Globar light source, passing through a $\mathrm{KBr}$ window. Raw photoconductivity spectra taken at 40 and $70 \mathrm{~K}$ are shown in

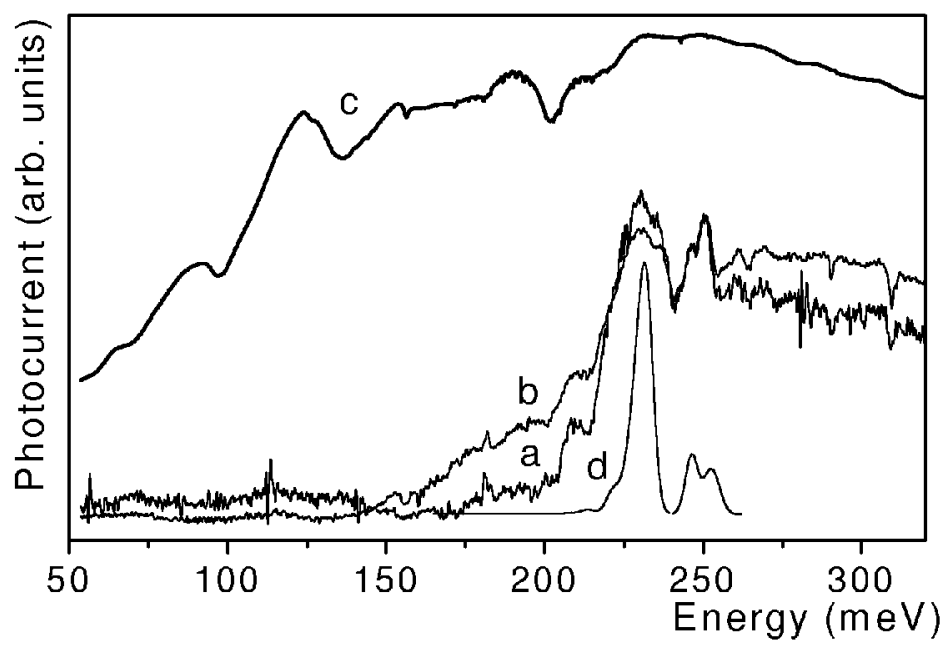

Fig. 1. Raw photoconductivity spectra taken at $40(a)$ and $70 \mathrm{~K}(b)$. The emission spectrum of the infrared light source is shown for comparison $(c)$. Trace $d$ shows the simulated photothermal ionization spectrum at $40 \mathrm{~K}$, taking $236 \mathrm{meV}$ as the ground state energy and a Gaussian line shape of $1 \mathrm{meV}$ width.

Fig. 1 (traces $a$ and $b$, respectively). The data were taken at low spectral resolution $\left(8 \mathrm{~cm}^{-1}\right)$ due to the weak signal. For comparison the spectrum of the infrared light source (with the $\mathrm{KBr}$ beam splitter) is shown (trace $c$ ). The photoconductivity data were not corrected for the spectral dependence of the light source due to the difficulty of filtering out the additional water vapor absorption in the $\mathrm{KBr}$ window.

\section{Theory}

The valence band in zinc-blende $\mathrm{GaN}$ is fourfold degenerate in the $\Gamma$ point of the Brillouin zone, which constitutes the common top of the heavy and light hole bands. The spin-orbit split-off band lies approximately $20 \mathrm{meV}$ below. The 


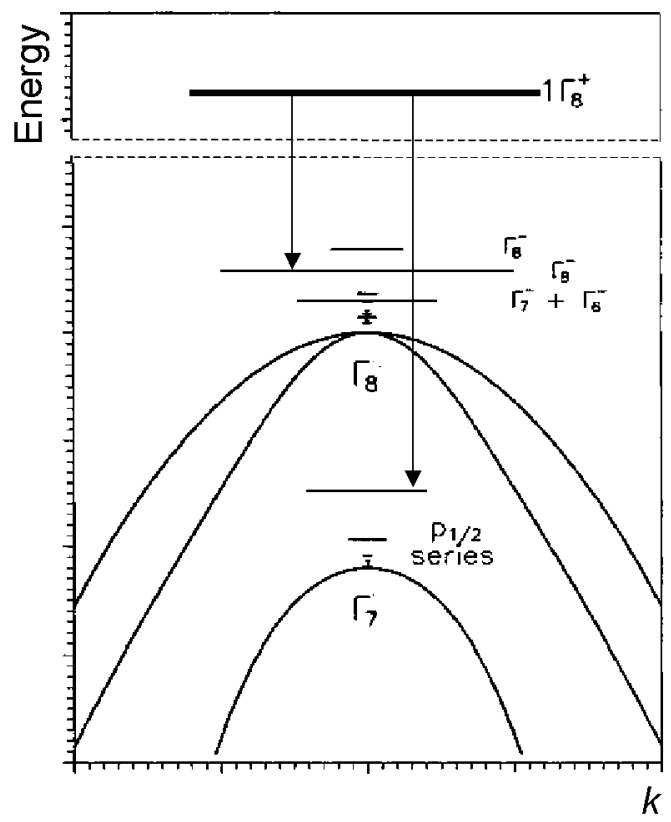

Fig. 2. Scheme of the acceptor states.

valence band structure is depicted schematically in Fig. 2. In order to calculate the shallow acceptor states the whole $6 \times 6$ Hamiltonian describing all these bands has to be used. Although the acceptor binding energies in GaN are quite high they are still much smaller than the band gap and the effective mass theory can be applied, especially for excited and resonant states. All states were calculated using the method described in the paper of Buczko and Bassani [2].

In the calculations the $6 \times 6$ Luttinger-Kohn [3] Hamiltonian in the spherical tensor representation [4] with effective mass parameters taken from Ref. [5] was adopted. In our model the potential of acceptor impurity is given by the Coulombic part screened by the dielectric constant and additional central cell correction term,

$$
U(r)=\frac{A}{r} \mathrm{e}^{-\beta r},
$$

responsible for the chemical shift of the ground state energy. Here $\beta=1$ a.u., and $A$ is an adjustable parameter. The trial envelope functions were expressed in terms of spherical harmonics and radial functions, and classified according to the irreducible representations of the $T_{d}$ group [2]. The radial functions were chosen of the form

$$
f_{l}=\sum_{i} c_{i} r^{l} \mathrm{e}^{-\alpha_{i} r},
$$

with $\alpha_{i}$ values covering a wide spectrum of length scales. Since the effective-mass Hamiltonian applied is invariant under inversion with respect to the origin, the 
envelope functions can be additionally classified as of even or odd parity. The ground state is of even parity and the parameter $A$ in Eq. (1) was adjusted in such a way that the ground state energy agrees with the value of $E=230 \mathrm{meV}$ estimated from photoluminescence experiments [6]. With effective-mass parameters taken from Ref. [5] we obtained $A=4.6$. In addition to the ground state we found oddand even-parity excited states, as well as resonant states. The latter are connected to the top of spin-orbit split-off band and are present in the energy region of the light and heavy hole bands (see Fig. 2). They were distinguished from spurious solutions using the same method as described in Ref. [2].

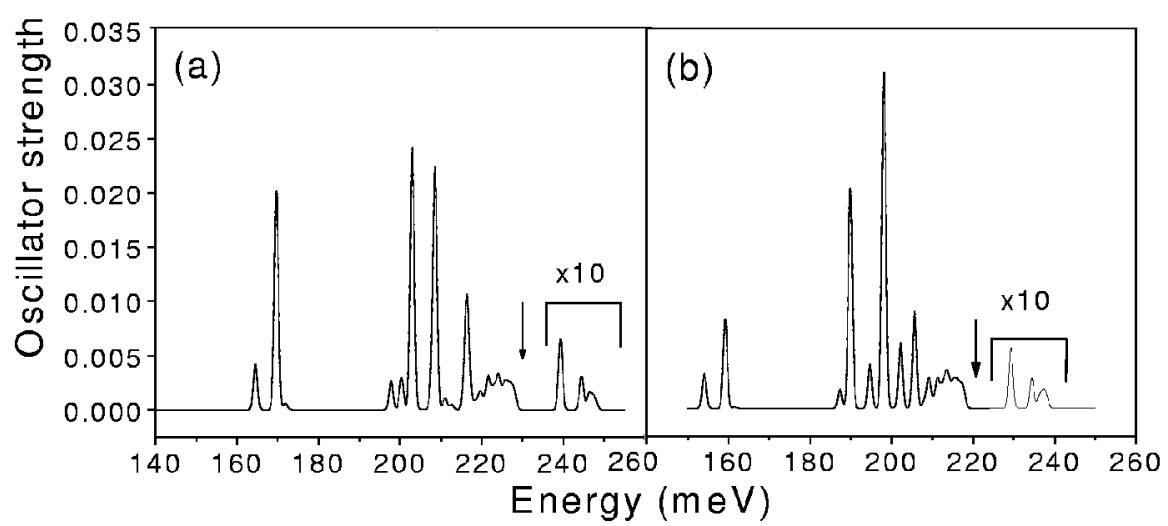

Fig. 3. Oscillator strengths of the optical transitions from the ground state (a) and the first even-parity excited state (b) to odd-parity excited and resonant states. A Gaussian line shape was assumed for every transition, with an amplitude given by the calculated oscillator strength and a width of $0.5 \mathrm{meV}$. The arrows mark the onset of the valence band continuum. The shift in the energy scale between cases (a) and (b) is due to the calculated spin-orbit splitting of the ground state $(10.4 \mathrm{meV})$.

Finally, the oscillator strengths of the optical transitions from the ground state and the first even-parity excited state (related to the spin-orbit split-off band) to the odd-parity excited and resonant states were evaluated by calculation of the appropriate matrix elements in the dipole approximation. The computed spectra are shown in Figs. 3a and b, respectively. In the figures a Gaussian line shape was assumed for every transition, with an amplitude given by the calculated oscillator strength and a width of $0.5 \mathrm{meV}$. In Fig. 3 only transitions to discrete states are shown. The arrows mark the position of the top of the valence band.

\section{Discussion}

In contrast to absorption measurements in photoconductivity experiments the intensity of the signal related to a particular internal transition depends not 
only on the oscillator strength but also on the thermal ionization energy from the final state to the valence band. At low temperatures, transitions to states lying close to the continuum or within the continuum can be, hence, more intense than those occurring at lower transition energies. In photostimulated current (Fig. 1) two relatively broad features are observed, centered at about 230 and $250 \mathrm{meV}$. The experimental peak positions are consistent with numerical calculations for the Mg-related transitions to odd-parity bound states and resonant states, respectively, provided that the $\mathrm{Mg}$ ground state energy is shifted from $230 \mathrm{meV}$ (assumed initially for the chemical shift calculations) to $236 \mathrm{meV}$, relative to the top of the valence band. Since the binding energies of odd parity states are only weakly influenced by central cell corrections, in contrast to the ground state energy that depends strongly on the choice of parameter $A$ in Eq. (1), a small shift in the ground-state energy should hardly affect the former.

The simulated photothermal ionization spectrum at $40 \mathrm{~K}$ is shown in Fig. 1 (trace $d$ ). Here, the line width was chosen to match the experimental spectral resolution and, again, only transitions to discrete states were included. The ground state energy was taken as $236 \mathrm{meV}$ and for the thermal ionization the calculated bound states energies relative to the top of the valence band were taken. It can be seen that the agreement between experimental data and calculations is quite satisfactory but for the line width. The experimental peaks are much broader, indicative of strain in the GaN layer. This is not unexpected due to lattice mismatch between GaN and GaAs, also precipitates of the wurtzite phase, observed in photoluminescence, could add locally to the overall strain and contribute to the broadening.

At lower energies an additional, weak photoionization band was observed (see Fig. 1), with an onset at $\approx 150 \mathrm{meV}$ (the apparent structure is entirely due to that of the IR light source). The onset agrees with the binding energy of a native acceptor, commonly found in zinc-blende GaN [6] and observed also in the photoluminescence of the sample studied. With increasing temperature the contribution of the native acceptor to the photocurrent increases, which might be caused by the difference in hole capture rates.

\section{Conclusions}

Internal transitions of the magnesium acceptor in zinc-blende GaN were observed for the first time in photoconductivity. The transitions were identified with the help of effective-mass model calculations involving light- and heavy-hole as well as spin-orbit split-off bands. Transitions to the states associated with the spin-orbit split-off valence band, superimposed on the photoionization band, were also observed. The hole binding energy of the $\mathrm{Mg}$ acceptor in cubic phase GaN was determined as $236 \pm 1 \mathrm{meV}$, close to the value estimated from photoluminescence. An unstructured photoionization band related to a native acceptor of $\approx 130 \mathrm{meV}$ binding energy was also detected. 


\section{Acknowledgments}

The authors are indebted to D.J. As and K. Lischka for providing the samples studied. This work was supported in part by a grant from the State Committee for Scientific Research, grant No. 5 P03B 12321.

\section{References}

[1] J.W. Orton, C.T. Foxon, Rep. Prog. Phys. 61, 1 (1998), and references therein.

[2] R. Buczko, F. Bassani, Phys. Rev. B 45, 5838 (1992).

[3] J.M. Luttinger, W. Kohn, Phys. Rev. 97, 869 (1955).

[4] A. Baldereschi, N.O. Lipari, Phys. Rev. B 8, 2697 (1973); Phys. Rev. B 9, 1525 (1974).

[5] K. Kim, W.R.L. Lambrecht, B. Segall, M. Van Schilfgaarde, Phys. Rev. B 56, 7363 (1997).

[6] D.J. As, T. Simonsmerier, B. Schöttker, T. Frey, D. Schikora, W. Kriegeis, W. Burkhardt, B.K. Meyer, Appl. Phys. Lett. 73, 1835 (1998). 